\title{
Inflammatory Myopathies
}

National Institute of Neurological Disorders and Stroke (NINDS)

\section{Source}

National Institute of Neurological Disorders and Stroke (NINDS). Inflammatory

Myopathies Information Page.

The inflammatory myopathies are a group of diseases, with no known cause, that involve chronic muscle inflammation accompanied by muscle weakness. The four main types of chronic, or long-term, inflammatory myopathies are polymyositis, dermatomyositis, inclusion body myositis, and necrotizing autoimmune myopathy. Although the cause of many inflammatory myopathies is unknown, the majority are considered to be autoimmune disorders, in which the body's immune response system that normally defends against infection and disease attacks its own muscle fibers, blood vessels, connective tissue, organs, or joints. These rare disorders may affect both adults and children, although dermatomyositis is more common in children. Polymyositis and dermatomyositis are more common in women than in men. Inclusion body myositis is most common after age 50. General symptoms of chronic inflammatory myopathy include slow but progressive muscle weakness that starts in the proximal musclesthose muscles closest to the trunk of the body. Other symptoms include fatigue after walking or standing, tripping or falling, and difficulty swallowing or breathing. Some individuals may have slight muscle pain or muscles that are tender to the touch. Polymyositis affects skeletal muscles (involved with making movement) on both sides of the body. Dermatomyositis is characterized by a skin rash that precedes or accompanies progressive muscle weakness. IBM is characterized by progressive muscle weakness and wasting. Juvenile myositis has some similarities to adult dermatomyositis and polymyositis. Symptoms of necrotizing autoimmune myopathy include weakness in both the upper and lower body, difficulty rising from low chairs, climbing stairs, or lifting objects, fatigue, weight loss, and muscle pain. 